Pacific Journal of Mathematics

ON THE BOUNDARY CURVES OF INCOMPRESSIBLE 


\title{
ON THE BOUNDARY CURVES OF INCOMPRESSIBLE SURFACES
}

\author{
A. E. HATCheR
}

Let $K$ be a knot in $S^{3}$, and consider incompressible (in the stronger sense of $\pi_{1}$-injective), $\partial$-incompressible surfaces $S$ in the exterior of $K$. A question which has been around for some time is whether the boundary-slope function $S \mapsto$ $m_{S} / \ell_{S}$, where $m_{S}$ and $\ell_{S}$ are the numbers of times each circle of $\partial S$ wraps around $K$ meridionally and longitudinally, takes on only finitely many values (for fixed $K$ ). This is known to be true for certain knots: torus knots, the figure-eight knot [4], 2-bridge knots [2], and alternating knots [3]. In this paper an affirmative answer is given not just for knot exteriors, but for all compact orientable irreducible 3-manifolds $M$ with $\partial M$ a torus. Further, we give a natural generalization to the case when $\partial M$ is a union of tori.

To state this more general result it is convenient to use the projective lamination space $\mathscr{P} \mathscr{L}(\partial M)$, defined in [4]. If $\partial M$ is the union of tori $T_{1}, \cdots, T_{n}$, then $\mathscr{P} \mathscr{L}(\partial M)$ is the join $\mathscr{P} \mathscr{L}\left(T_{1}\right) * \cdots *$ $\mathscr{P} \mathscr{L}\left(T_{n}\right)=\boldsymbol{R} P^{1} * \cdots * \boldsymbol{R} P^{1}$, a sphere $S^{2 n-1}$. More concretely, suppose coordinates are chosen for each $T_{i}$. Then isotopy classes of finite systems of disjoint noncontractible simple closed curves on $T_{i}$ are parametrized by the set $\boldsymbol{Z}^{2} / \pm$ of pairs $(a, b) \in \boldsymbol{Z}^{2}$, where $(a, b)$ is identified with $(-a,-b)$. So systems on $\partial M$ are parametrized by $\left(\boldsymbol{Z}^{2} / \pm\right)^{n}$. Restricting to nonempty systems and projectivising by identifying a system with any number of parallel copies of itself, yields $\left(\boldsymbol{Z}^{2} / \pm\right)^{n}-\{0\} /(v \sim \lambda v)$. This is the same as $\left(\boldsymbol{Q}^{2} / \pm\right)^{n}-\{0\} /(v \sim \lambda v)$. The natural completion of this space is $\mathscr{P} \mathscr{L}(\partial M)=\left(\boldsymbol{R}^{2} / \pm\right)^{n}-\{0\} /$ $(v \sim \lambda v)$, clearly a sphere of dimension $2 n-1$. (We shall not be concerned with the geometrical interpretation of the points added in forming this completion.) A change of coordinates for the $T_{i}$ 's produces a projective transformation of this $S^{2 n-1}$, so $\mathscr{P} \mathscr{L}(\partial M)$ has a natural projective structure. (For surfaces of higher genus, $\mathscr{P} \mathscr{L}$ has only a natural piecewise projective structure.)

TheOREM. Let $M$ be orientable, compact, irreducible, with $\partial M$ a union of $n$ tori. Then the projective classes of curve systems in $\partial M$ which bound incompressible, $\partial$-incompressible surfaces in $M$ form a dense subset of a finite (projective) polyhedron in $\mathscr{P} \mathscr{L}(\partial M)=S^{2 n-1}$ of dimension less than $n$.

CoRollary. If $\partial M=T^{2}$, there are just a finite number of slopes 
realized by boundary curves of incompressible, $\partial$-incompressible surfaces in $M$.

Returning to the case of a knot $K \subset S^{3}$, this means that if the peripheral torus $\partial N(K)$ is the only closed incompressible surface in $S^{3}-K$, up to isotopy, then all but a finite number of Dehn surgeries on $K$ yield irreducible non-Haken manifolds. More generally, for links $L \subset S^{3}$ of $n$ components such that $S^{3}-L$ is irreducible (i.e., $L$ is nonsplit) and contains no closed nonperipheral incompressible surfaces, the theorem implies that the coefficients $\left(p_{1} / q_{1}, \cdots, p_{n} / q_{n}\right) \in T^{n}$ of the Dehn surgeries on $L$ which yield either nonirreducible or Haken manifolds lie in a piecewise smooth finite subcomplex of $T^{n}$ of dimension less than $n$. Thus, "most" surgeries on $L$ yield irreducible non-Haken manifolds.

One may compare the assertion of the theorem with the fact (a consequence of duality) that the image of the boundary map $H_{2}(M, \partial M) \rightarrow H_{1}(\partial M)$ has rank equal to half the rank of $H_{1}(\partial M)$. Thus, passing to the sphere $S^{2 n-1}$ of rays through the origin in $H_{1}(\partial M ; R)$, the image of the homological boundary map is a sphere $S^{n-1} \subset S^{2 n-1}$.

The proof of the theorem will follow fairly easily from a recent fundamental result of [1] about branched surfaces in 3-manifolds, i.e., closed subsets locally diffeomorphic to the model in Figure 1a.

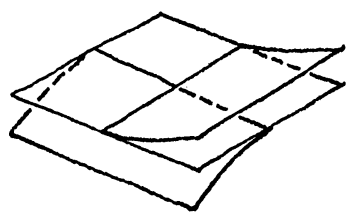

(a)

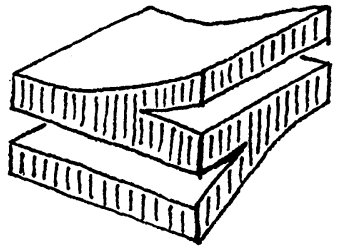

(b)

FIGURE 1

A branched surface $B$ is said to carry a surface $S$ if $S$ lies in a fibered regular neighborhood $N(B)$ of $B$ (indicated in Figure 1b) and is transverse to all the fibers of $N(B) ; S$ has positive weights if it meets all the fibers of $N(B)$. The result of [1] is that in a compact irreducible 3-manifold $M$ with incompressible boundary there exist a finite number of branched surfaces $\left(B_{i}, \partial B_{i}\right) \subset(M, \partial M)$ such that the surfaces carried by these $B_{i}$ 's with positive weights are exactly all the incompressible, $\partial$-incompressible surfaces in $M$, up to isotopy.

Let $B \subset M$ be one of the branched surfaces of [1]. Then $\partial B=$ $B \cap \partial M$ is a branched 1-manifold in $\partial M$ with two key properties:

(1) There is no smooth disk $D \subset \partial M$ with $D \cap \partial B=\partial D$. 
(2) There is no disk $D \subset \partial M$, smooth except for one outward cusp point in $\partial D$, with $D \cap \partial B=\partial D$.

Condition (2) is explicitly given in [1]. If condition (1) failed, then any surface carried by $B$ with positive weights would have a boundary circle which was contractible in $\partial M$. This circle would then bound a disk component of the (incompressible) surface, contrary to the construction of $B$ in [1].

(In the terminology of [4], $\partial B$ would be a train track in $\partial M$, were it not for the fact that some components of $\partial M-\partial B$ can be digons.)

Let $S$ be a surface carried by $B$ with positive weights. No component of $\partial S$ can be contractible in $\partial M$, since otherwise there would be a smooth disk $D \subset \partial M$ with $\partial D \subset \partial B$, and somewhere inside this disk condition (1) or (2) would be violated. Thus in each component $T_{i}$ of $\partial M$ which $B$ meets, $\partial S$ consists of a number of parallel nontrivial circles.

LEMma. There is an orientation $\mathcal{O}$ on $\partial B$ with the property that all the circles of $T_{i} \cap \partial S$, with the orientations induced from $\mathcal{O}$, are homologous in $T_{i}$. Hence the O-oriented class $[\partial S] \in H_{1}(\partial M)$ determines the class of $\partial S$ in $\mathscr{P} \mathscr{L}(\partial M)$, by simply forgetting the orientation and then projectivising.

Proof. Let $S_{0}$ be a surface carried by $B$ with positive weights. We can construct a fibered regular neighborhood $N\left(\partial S_{0}\right)$ of $\partial S_{0}$ in $\partial M$ from a fibered regular neighborhood $N(\partial B)$ of $\partial B$ in $\partial M$ by slitting $N(\partial B)$ along certain circles and arcs in $N(\partial B)$ transverse to the fibers. Inverting this construction, we see that $N(\partial B)$ is obtainable from $N\left(\partial S_{0}\right)$ by adding certain fibered rectangles and annuli in $\partial M-N\left(\partial S_{0}\right)$, as shown in Figure 2. (Annuli would be necessary if $\partial S_{0}$ contained pairs of circles which were parallel in $N(\partial B)$.)

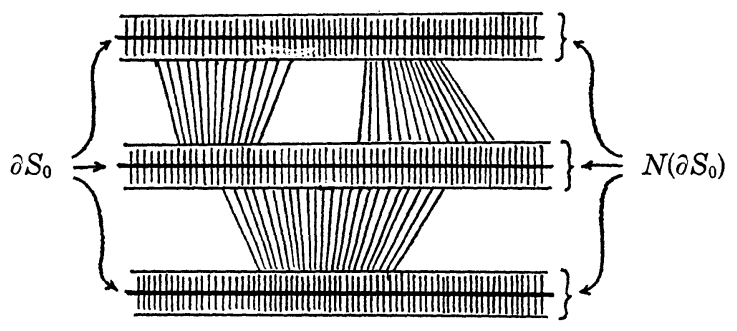

Figure 2

No fiber of a rectangle of $N(\partial B)-N\left(\partial S_{0}\right)$ can join a component of $\partial N\left(\partial S_{0}\right)$ to itself, since this would violate condition (2) above. Hence all the fibers of $N(\partial B)$ can be coherently oriented, as follows. On 
each component $T_{i}$ of $\partial M$ which $\partial B$ meets, choose parallel orientations for all the circles of $\partial S_{0} \cap T_{i}$; then an orientation for $T_{i}$ determines an orientation for the fibers of $N\left(\partial S_{0}\right) \cap T_{i}$ which extends to $N(\partial B) \cap T_{i}$.

We may choose an oriented simple closed curve $\gamma_{i}$ in $T_{i}$ meeting $N(\partial B)$ in union of fibers of $N(\partial B)$, such that the orientation of $\gamma_{i}$ agrees with the orientation of the fibers. To construct such a $\gamma_{i}$, start with any fiber of $N(\partial B)$, continue across a rectangle or annulus of $T_{i}-N(\partial B)$ to another fiber of $N(\partial B)$ on the opposite side of this rectangle or annulus, and so on. Eventually the curve so constructed must either close up or come arbitrarily close to closing up, in which case by rechoosing part of the curve in $T_{i}-N(\partial B)$ we can make it close up. From the existence of $\gamma_{i}$ the first statement of the lemma clearly follows, since all the points of $\gamma_{i} \cap \partial S$ have intersection numbers of the same sign. The second statement of the lemma is then immediate.

Proof of the Theorem. The surfaces carried by $B$ are determined by assigning nonnegative integer weights to the components of $B-B^{\prime}$, where $B^{\prime}$ is the branching locus of $B$. These weights $a_{i}$ must satisfy certain equations of the form $a_{i}+a_{j}=a_{k}$, coming from the branching at $B^{\prime}$. Thus the projective classes of surfaces carried by $B$ correspond to the "rational" points of a convex polyhedral cell which is the intersection of the $(N-1)$-simplex $[0, \infty)^{N}-$ $\{0\} /(v \sim \lambda v)$ with a linear subspace of $\boldsymbol{R}^{N}$, for some $N$. The same is true if one restricts to projective classes of boundary curve systems of surfaces carried by $B$ : they are parametrized by the rational points of a convex polyhedral cell, $c_{B}$ say. Restricting to surfaces of positive weights corresponds to taking $c_{B}$ to be an open cell. Moreover, by the lemma, the open cell $c_{B}$ maps into $\mathscr{P} \mathscr{L}(\partial M)$ by a projective linear map. (Regarding $\partial B$ as an oriented 1-complex, then a weighting of its edges by integers $a_{i}$ satisfying the branching conditions $a_{i}+a_{j}=a_{k}$ determines a 1-cycle, which obviously depends linearly on the $a_{i}$ 's.)

It remains to see that $\operatorname{dim} c_{B}<n$. Let $S_{1}$ and $S_{2}$ be two surfaces carried by $B$ with positive weights. If $\partial S_{1}$ and $\partial S_{2}$ are oriented by $O$, then the intersection number $\partial S_{1} \cdot \partial S_{2}$ is zero, provided $\partial M$ is oriented as the boundary of $M$. To see this, perturb $S_{1}$ and $S_{2}$ slightly to be transverse (and still transverse to the fibers of $N(B)$ ). There are two possible configurations for the $\mathcal{O}_{\text {-orientations of } \partial S_{1}}$ and $\partial S_{2}$ at the ends of an arc $\alpha$ of $S_{1} \cap S_{2}$, as shown in Figure 3 below (where the fibers of $N(B)$ are in the vertical direction). In either case the two ends of $\alpha$ give points of $\partial S_{1} \cap \partial S_{2}$ with opposite intersection numbers. So $\partial S_{1} \cdot \partial S_{2}=0$. 
Thus, the classes $\partial S$ for surfaces $S$ carried by $B$ with positive weights belong to a self-annihilating subspace of $H_{1}(\partial M) \approx Z^{2 n}$. By linear algebra, this subspace has rank $\leqq n$, and the theorem follows.
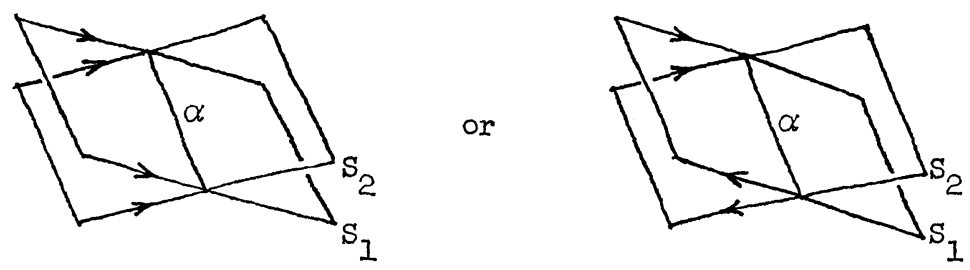

FIGURe 3

Questions.

(1) Is there a generalization of the theorem to 3-manifolds having boundary components of higher genus?

(2) For knot exteriors in $S^{3}$, must the boundary slopes of incompressible, $\partial$-incompressible surfaces always be integers?

(3) Are there nontrivial knots for which all the incompressible, $\partial$-incompressible surfaces have the same boundary slope?

\section{REFERENCES}

1. W. Floyd and U. Oertel, Incompressible surface via branched surfaces, to appear.

2. A. Hatcher and W. Thurston, Incompressible surfaces in 2-bridge knot complements, to appear.

3. W. Menasco, Incompressible surfaces in the complement of alternating knots and links, preprint.

4. W. Thurston, Geometry and topology of 3-manifolds, to appear.

Received October 15, 1980 and in revised form December 2, 1980.

UNIVERSITY OF CALIFORNIA

LOS ANgeles, CA 90024 



\title{
PACIFIC JOURNAL OF MATHEMATICS
}

\section{EDITORS}

\author{
DONALD BABBITT (Managing Editor) \\ University of California \\ Los Angeles, CA 90024 \\ Hugo RossI \\ University of Utah \\ Salt Lake City, UT 84112 \\ C. C. MOORE and Arthur Agus \\ University of California \\ Berkeley, CA 94720
}

\section{J. DugundjI}

Department of Mathematics

University of Southern California

Los Angeles, CA 90007

R. FinN and J. Milgram

Stanford University

Stanford, CA 94305

\section{ASSOCIATE EDITORS}
R. ARENS
E. F. BeCKENBACH
B. H. NeumanN
F. WOLF
K. YoshidA

\section{SUPPORTING INSTITUTIONS}

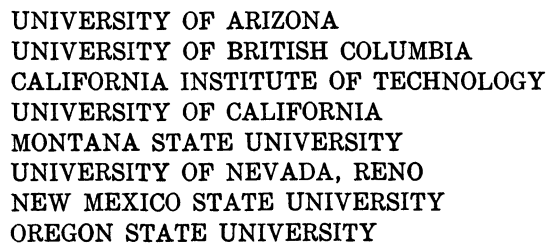

UNIVERSITY OF ARIZONA

UNIVERSITY OF BRITISH COLUMBIA

CALIFORNIA INSTITUTE OF TECHNOLOGY

UNIVERSITY OF CALIFORNIA

MONTANA STATE UNIVERSITY

UNIVERSITY OF NEVADA, RENO

NEW MEXICO STATE UNIVERSITY

OREGON STATE UNIVERSITY

\author{
UNIVERSITY OF OREGON \\ UNIVERSITY OF SOUTHERN CALIFORNIA \\ STANFORD UNIVERSITY \\ UNIVERSITY OF HAWAII \\ UNIVERSITY OF TOKYO \\ UNIVERSITY OF UTAH \\ WASHINGTON STATE UNIVERSITY \\ UNIVERSITY OF WASHINGTON
}

The Supporting Institutions listed above contribute to the cost of publication of this Journal, but they are not owners or publishers and have no responsibility for its content or policies.

Mathematical papers intended for publication in the Pacific Journal of Mathematics should be in typed form or offset-reproduced, (not dittoed), double spaced with large margins. Please do not use built up fractions in the text of the manuscript. However, you may use them in the displayed equations. Underline Greek letters in red, German in green, and script in blue. The first paragraph or two must be capable of being used separately as a synopsis of the entire paper. Please propose a heading for the odd numbered pages of less than 35 characters. Manuscripts, in triplicate, may be sent to any one of the editors. Please classify according to the scheme of Math. Reviews, Index to Vol. 39. Supply name and address of author to whom proofs should be sent. All other communications should be addressed to the managing editor, or Elaine Barth, University of California, Los Angeles, California, 90024.

50 reprints to each author are provided free for each article, only if page charges have been substantially paid. Additional copies may be obtained at cost in multiples of 50 .

The Pacific Journal of Mathematics is issued monthly as of January 1966. Regular subscription rate: $\$ 102.00$ a year (6 Vols., 12 issues). Special rate: $\$ 51.00$ a year to individual members of supporting institutions.

Subscriptions, orders for numbers issued in the last three calendar years, and changes of address shoud be sent to Pacific Journal of Mathematics, P.O. Box 969, Carmel Valley, CA 93924, U.S.A. Old back numbers obtainable from Kraus Periodicals Co., Route 100, Millwood, NY 10546.

\section{PUBLISHED BY PACIFIC JOURNAL OF MATHEMATICS, A NON-PROFIT CORPORATION}

Printed at Kokusai Bunken Insatsusha (International Academic Printing Co., Ltd.). 8-8, 3-chome, Takadanobaba, Shinjuku-ku, Tokyo 160, Japan. 


\section{Pacific Journal of Mathematics}

Vol. 99, No. $2 \quad$ June, 1982

Thomas E. Armstrong and Karel Libor Prikry, On the semimetric on a

Boolean algebra induced by a finitely additive probability measure . . . .249

Walter Russell Bloom, Strict local inclusion results between spaces of

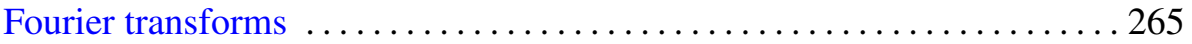

Richard Clark Brown, Notes on generalized boundary value problems in

Banach spaces. II. Infinite-dimensional extension theory ........... 271

Sui Sun Cheng, Isoperimetric eigenvalue problem of even order differential

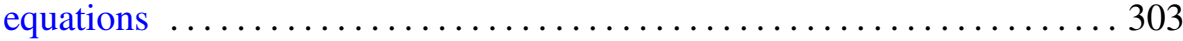

Lung O. Chung and Jiang Luh, Derivations of higher order and

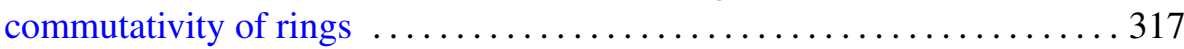

Ali Ahmad Fora, A fixed point theorem for product spaces . . . . . . . . . 327

Barry J. Gardner, Radical classes of regular rings with Artinian primitive

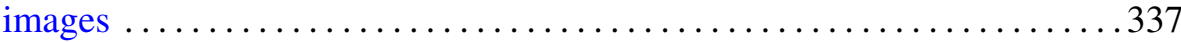

John Brady Garnett and Peter Wilcox Jones, BMO from dyadic BMO . . . 351

Allen E. Hatcher, On the boundary curves of incompressible surfaces . . . . 373

Richard Howard Hudson and Kenneth S. Williams, Resolution of

ambiguities in the evaluation of cubic and quartic Jacobsthal sums .....379

Viktor Losert, Counter-examples to some conjectures about doubly

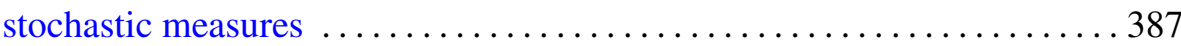

Kenneth Derwood Magill, Jr., P. R. Misra and Udai Bhan Tewari,

Structure spaces for sandwich semigroups

Mark Mandelker, Continuity of monotone functions

Kenneth Guy Miller, An index theorem and hypoellipticity on nilpotent Lie groups ......................................... 419

Evelyn M. Nelson, Homomorphisms of mono-unary algebras . . . . . . . . . 427

Marvin E. Ortel, The support of an extremal dilatation . . .

R. S. Pathak and O. P. Singh, Finite Hankel transforms of distributions . . . 439

Richard Cole Penney, The theory of ad-associative Lie algebras

Linda Ruth Sons, Zero distribution of functions with slow or moderate

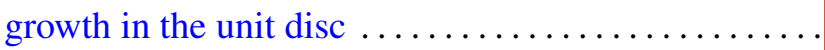

Russell Bruce Walker, Transversals to laminations 